\title{
Otto-coupled surface plasmons in a liquid crystal cell
}

\author{
Lizhen Ruan, ${ }^{\text {a) }}$ Fuzi Yang, and J. R. Sambles \\ Electro-Magnetic Materials, School of Physics, University of Exeter, Exeter EX4 4QL, United Kingdom
}

(Received 10 July 2009; accepted 11 September 2009; published online 26 October 2009)

\begin{abstract}
Surface plasmons on silver in the visible domain are excited using a several micron thick layer of liquid crystal as the optical tunnel barrier. This thickness is possible when the orientation of the director in the liquid crystal varies from homeotropic at the entrance surface (against the coupling prism) to homogeneous on the thick silver layer at the other side of the cell, with the director tilting in a plane normal to the plane of incidence. This geometry also allows the excitation of guided modes, which mixes with the surface plasmon resonance. Both types of mode are then explored as a function of applied voltage. () 2009 American Institute of Physics. [doi:10.1063/1.3242363]
\end{abstract}

Surface plasmon polaritons $(\mathrm{SPPs})^{1}$ are waves that are localized to and propagate along the surface of a conductor, usually a metal. For a planar surface solving Maxwell's equations under the appropriate boundary conditions one obtains the simple SPP dispersion relation

$$
k_{s p}=k_{0} \sqrt{\frac{\varepsilon_{d} \varepsilon_{m}}{\varepsilon_{d}+\varepsilon_{m}}},
$$

where $\varepsilon_{d}$ and $\varepsilon_{m}$ are the permittivities of the dielectric contacting with the metal layer, and the metal, respectively. If the real parts of $\varepsilon_{d}$ and $\varepsilon_{m}$ are of opposite sign and $\left|\varepsilon_{m}\right|$ $>\varepsilon_{d}$, this may give a trapped surface wave, the SPP, the in-plane momentum of which is larger than a plane electromagnetic wave of the same frequency in the adjacent dielectric. Due to this momentum mismatch, for incident radiation to couple to the SPP some form of momentum enhanced coupling technique must be introduced. In the case of using a coupling prism to provide this extra momentum there are two different geometries, the Otto ${ }^{2}$ and the Kretschmann-Raether geometries. ${ }^{3}$ For the exploration of the near-surface director profile in liquid crystals several researchers have used the optical excitation of $\mathrm{SPPs}^{4-6}$ with the Kretschmann-Raether geometry in which a thin metal film (often silver) acts as the optical tunnel barrier. The Otto geometry is perceived as being difficult to use because it normally requires a dielectric thickness of the order of $0.5 \mu \mathrm{m}$ in the visible domain.

In this study the Otto geometry is used in a rather thick liquid crystal (LC) cell with a particular director profile, the LC itself acting as the tunnel barrier. When the orientation of the director in the LC cell varies from homeotropic at the entrance surface (against the coupling prism) to homogeneous on a thick silver layer at the other side of the cell, an Otto-type SPP mode may be excited even for quite thick cells (several microns). This half-leaky guided mode (HLGM) geometry ${ }^{7}$ is then used to explore the distribution of the LC director near to the silver surface and the effects of different cell thicknesses and applied electrical fields on the SPP and guided modes are explored.

A LC cell of $\sim 8 \mu \mathrm{m}$ thickness is assembled using two glass plates. One is a high index plate $(n=1.732$ at $632.8 \mathrm{~nm}$ ), on which there is a thin electrode layer of indium tin oxide on the inner surface, on top of which is a lecithin aligning layer. On to the bottom plate is deposited an opti-

${ }^{a)}$ Electronic mail: 1.ruan@exeter.ac.uk. cally opaque $(200 \mathrm{~nm})$ silver layer on which is a rubbed polyimide (PI) layer to provide homogeneous alignment. This cell is filled with the nematic liquid crystal E7 (from Merck). Thus the orientation of the director in the cell varies from homeotropic at the top (entrance face) of the cell to homogeneous alignment on the silver surface forming a hybrid aligned nematic (HAN) cell.

In order to excite the SPPs and the related guided modes, the half-leaky guided wave technique ${ }^{7}$ is used. The cell is inserted between a high index $(n=1.732$ at $632.8 \mathrm{~nm})$ prism and a glass block. A high index matching fluid $(n=1.732$ at $632.8 \mathrm{~nm}$ ) is used to make optical contact of the top high index glass plate of the cell with the prism. The glass block is simply used to support the cell during the measurement. The experimental setup is a typical $\theta-2 \theta$ rotating system as described elsewhere ${ }^{8}$ with the cell set so that the rubbing direction of the PI layer is perpendicular to the incident plane. A plane polarized $p$ (or $s$ ) beam with $\lambda=632.8 \mathrm{~nm}$ (He-Ne laser) enters through one face of the input prism and the incident angle dependent reflectivity signals are recorded using a detector with $p$ - (or $s$ )-polarizer placed in front of it. Data in the form of $R_{p p}$ and $R_{s s}$ are recorded with various voltages applied to the cell at a frequency of $1 \mathrm{kHz}$. With different pressures applied to the glass block on the back of the cell, data for different thicknesses of the cell (and the same surface treatment) may be recorded. An SPP mode is found even with cells of thickness of order $8 \mu \mathrm{m}$ and it is found to be sensitive to changes in the cell thickness and the applied voltage. Typical $R_{p p}$ and $R_{s s}$ signals, for a $5.5 \mu \mathrm{m}$ thick cell with no applied voltage, are shown by the crosses in Fig. $1 . R_{p p}$ is a $p$-polarized reflectivity against $p$-polarized incoming beam and $R_{s s}$ is an $s$-polarized reflectivity against $s$-polarized incoming beam.

Using multilayer optics theory, together with a continuum elastic theory for the nematic such reflectivity data are fitted to a model giving thereby the full director profiles within the cell at different thicknesses and different applied voltages. For no applied field the director tilt varies linearly from homeotropic at the high index plate to nearhomogeneous with a small pretilt angle on the silver surface. By fitting the data the optical parameters of the LC E7 are found to be $\varepsilon_{\perp}=2.3030+i 0.0001$ and $\varepsilon_{\|}=3.0250+i 0.0001$. Note that the reflectivity data $R_{S s}$ are used for determining the cell thickness. Owing to the SPP mode cannot be excited by an $s$-polarized incoming beam, thus the spectrum of $R_{s s}$ 


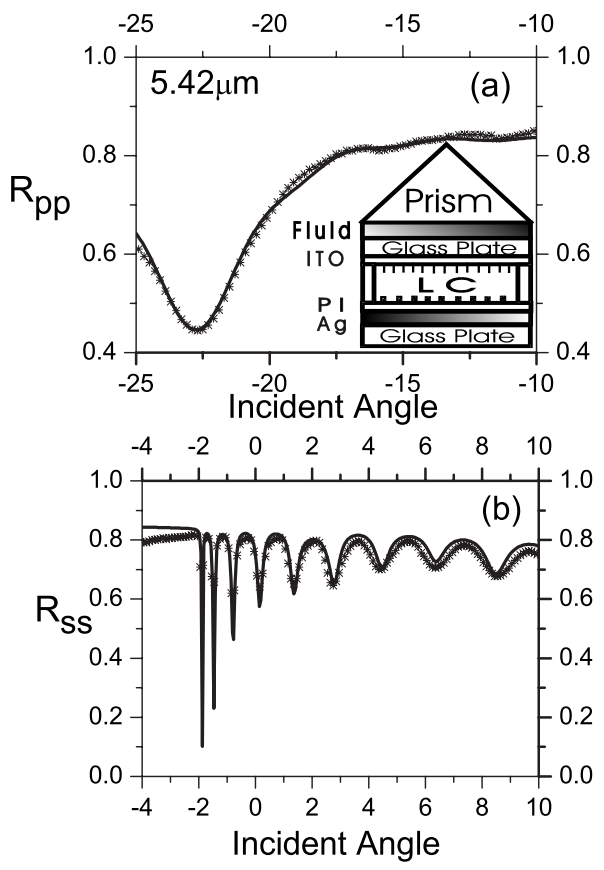

FIG. 1. Experimental data (crosses) (a) $R_{p p}$ and (b) $R_{s s}$ for a $5.5 \mu \mathrm{m}$ thick cell compared with the theoretical fits (solid lines). Inset is the cell structure.

with regular guided mode style [see Fig. 1(b)] can be easily used to determine the cell thickness.

The experiment shows very clearly that in this geometry the SPP, using $p$-polarized incident radiation, may be excited on the bottom silver surface using the Otto method for a tunnel barrier which is several wavelengths thick. This use of such a thick layer is allowed because at the incident surface the homeotropic director provides a high momentum for the $p$-polarized incident beam, which mainly depends on the optical parallel permittivity $\varepsilon_{\|}$of the liquid crystal material,
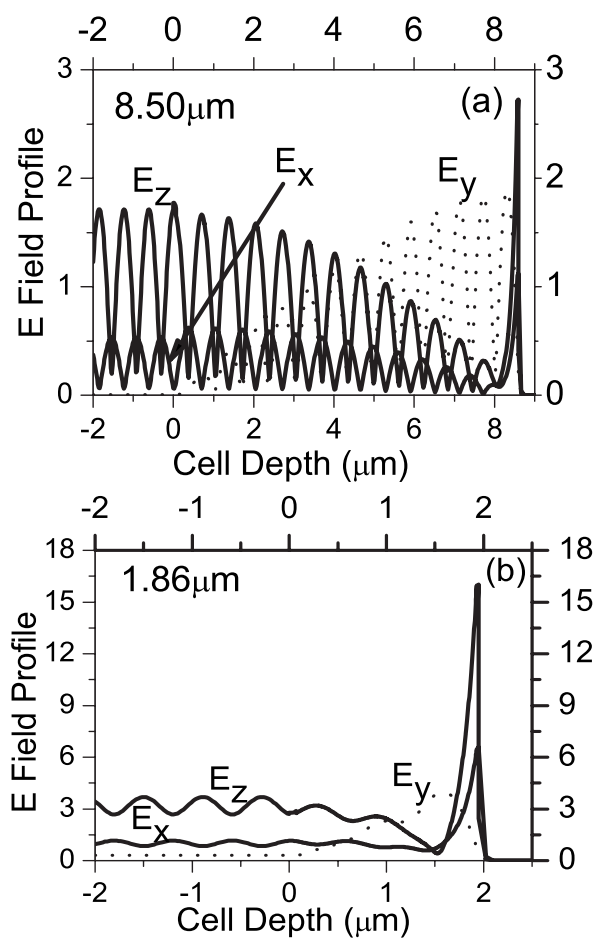

FIG. 2. Optical electric field profiles for different cell thicknesses (a) $8.5 \mu \mathrm{m}$ and (b) $1.86 \mu \mathrm{m}$.

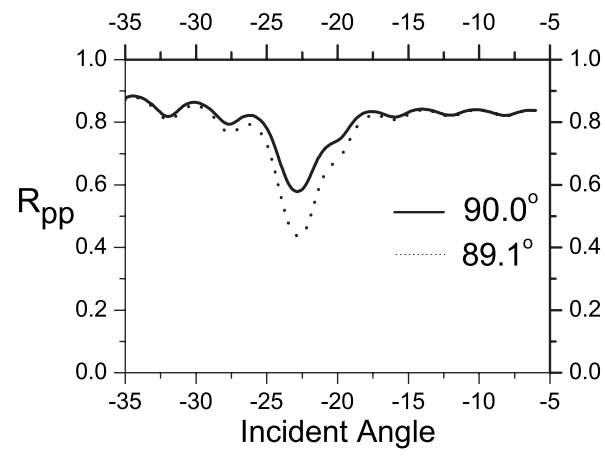

FIG. 3. The effect of director pretilt on the SPP mode.

while the momentum required for exciting the SPP on the bottom silver surface is primarily determined by the perpendicular permittivity $\varepsilon_{\perp}$. For E7 $\varepsilon_{\|}$is larger than $\varepsilon_{\perp}$, so the large in-plane momentum at the top surface transfers through the liquid crystal cell to the bottom, and at a certain incidence angle it matches the SPPs momentum on the bottom silver surface and a SPP resonance appears as a reflectivity minimum. The optical electrical field profile in the cell at this incident angle is shown in Fig. 2 for different thicknesses. Notice that the incident $p$-polarization (TM described by $E_{z}$ and $E_{x}$ ) gradually decreases in magnitude from the top of the cell to the bottom, as it is partly converted to $s$-polarization $\left(E_{y}\right)$ due to the director configuration in the HAN cell. A certain amount of TM field remains to excite a SPP on the silver surface. From modeling the data it is found that the strength of the SPP mode is very sensitive to the director orientation near to the silver surface. A change in only $1^{\circ}$ in director tilt angle at the bottom of a $5.5 \mu \mathrm{m}$ cell causes a change in more than $20 \%$ in the depth of the SPP mode (see Fig. 3). So this Otto coupling SPP geometry may be used to quantify the director pretilt on the silver surface. In our case a $1.2^{\circ}$ director pretilt angle on the rubbed PI layer has been determined from the fit to the data of Fig. 1(a).

By applying pressure to the glass block supporting the cell the cell thickness changes and results for different cell thicknesses are shown by the crosses in Fig. 4. The coupling strength to the SPP mode increases with decreasing cell thickness with the $p$-polarized electrical field profiles in the cell for different thickness being shown in Fig. 2. It is apparent that the thinner the cell the greater the transfer of energy to the surface plasmon.

However the effect of different applied voltages on the SPP mode is rather more complicated. On increasing the applied electrical field the SPP mode first becomes stronger, until, above a critical voltage, the SPP mode gradually fades

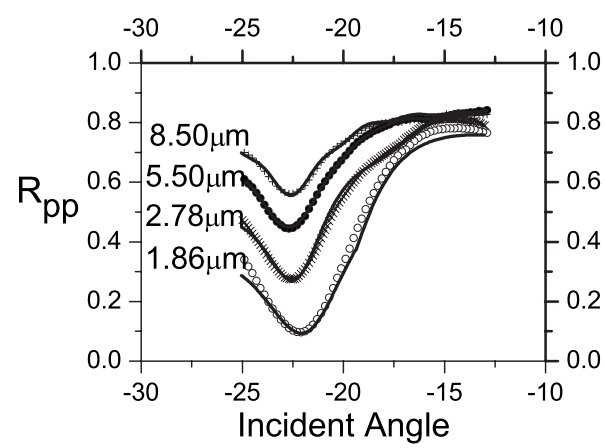

FIG. 4. Changes in the SPPs with different cell thicknesses. 


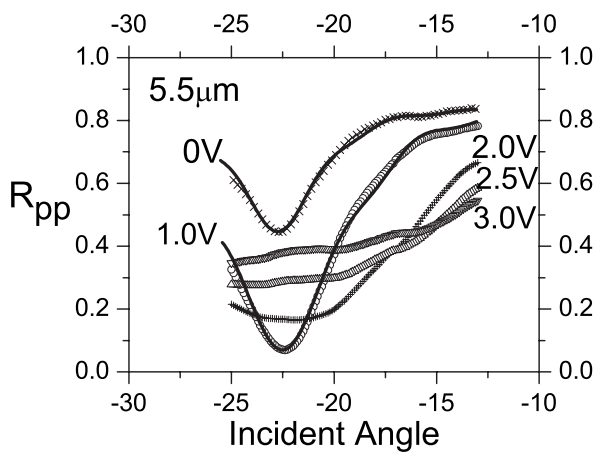

FIG. 5. Changes in the SPPs with different applied voltages for a $5.5 \mu \mathrm{m}$ cell.

until eventually there is no apparent SPP mode. Figure 5 shows the change in the SPP mode with different applied voltages for a $5.5 \mu \mathrm{m}$ thick cell, for which the critical voltage is about $1.0 \mathrm{~V}$. This critical voltage, for the excitation of the deepest SPP resonance, depends on the cell thickness. The reason for this effect may be explained as follows. At low applied fields the director near the top of the cell will align more perpendicular to the cell surface. Thus the $p$-polarized beam, entering from the top of the cell, may propagate further into the cell. That means that there is more guided mode energy reaching the bottom and exciting a stronger SPP mode. Of course eventually the system becomes overcoupled and, as is obvious from the data of Fig. 5 , the SPP resonance shallows and broadens. Furthermore note that on continued increase in the applied voltage such that the director in most of the cell is nearly homeotropic the conversion from $p$ - to $s$-polarization becomes very weak and the surface plasmon also moves out to much higher momentum with strong broad $p$-polarized guided modes resonances being recorded.

In conclusion, using a HAN liquid crystal cell (E7) with an opaque $(200 \mathrm{~nm})$ thick silver layer on the bottom surface of the cell an Otto-type SPP mode has been excited with a very thick (several microns) dielectric tunnel barrier. By using the optical HLGM technique the excitation of the SPP has been studied for different cell thicknesses and applied voltages. It is found that this Otto-type SPP resonance is very sensitive to the surface director tilt near the silver wall. Coupling to the SPP strength increases, as expected, with decrease in cell thickness. Applying different voltages to the cell also strongly influences this coupling, there being an optimum voltage to produce the strongest coupling to the SPP mode. Model optical electric field profiles within the cell help to explain these results and confirm that this modified geometry provides a powerful tool for exploration of near-surface director studies.

The authors are grateful to the Engineering and Physical Sciences Research Council for support under Grant No. EP/ D055652/1.

${ }^{1}$ R. H. Ritchie, Phys. Rev. 106, 874 (1957)

${ }^{2}$ A. Otto, Z. Phys. 216, 398 (1968).

${ }^{3}$ E. Kretschmann and H. Reather, Z. Naturforsch. A 23A, 2135 (1968)

${ }^{4}$ G. J. Sprokel, R. Santo, and J. D. Swalen, Mol. Cryst. Liq. Cryst. 68, 29 (1981).

${ }^{5}$ K. R. Welford and J. R. Sambles, J. Mod. Opt. 35, 1467 (1988).

${ }^{6}$ S. J. Elston, J. R. Sambles, and M. G. Clark, J. Mod. Opt. 36, 1019 (1989).

${ }^{7}$ F. Yang and J. R. Sambles, J. Opt. Soc. Am. B 10, 858 (1993).

${ }^{8}$ F. Yang and J. R. Sambles, J. Opt. Soc. Am. B 16, 488 (1999). 